\title{
Acknowledgement of reviewers for volume 33
}

(C) Australasian College of Physical Scientists and Engineers in Medicine 2010

The journal is deeply indebted to the reviewers who generously donate their time and expertise to evaluate and improve manuscripts. The names of those reviewers who dealt with manuscripts submitted for consideration for volume 33 are given below:

Rocio Alba-Flores (USA)

Barry Allen (Australia)

Will Ansbacher (Canada)

Mike Bailey (Australia)

Leighton Barnden (Australia)

Alun Beddoe (UK)

Bryan Bednarz (USA)

Phil Bones (New Zealand)

Bachir Boucheham (Algeria)

John Burrage (Australia)

Robert Chambers (Australia)

Varun Chandola (USA)

Vinod Chandran (Australia)

James Chow (Canada)

C. L. Chu (China)

Iwan Cornelius (Australia)

Dean Cutajar (Australia)

Weizhong Dai (USA)

Andrew Darling (USA)

Claire Dempsey (Australia)

M Descovich (USA)

Slobodan Devic (Canada)

Barbara Dobler (Germany)

Nesrin Dogan (USA)

Lawrence Dougherty (USA)

Lisa Duggan (Australia)

Martin Ebert (Australia)

Komiti Ese Enari (Australia)

Andrew Fielding (Australia)

Christopher Fox (Australia)
Peter Greer (Australia)

Jim Hagekyriakou (Australia)

Hugh Harris (Australia)

Joan Hatton (Australia)

Jens Haueisen (Germany)

Annette Haworth (Australia)

Jari O. Heikkinen (Finland)

Robin Hill (Australia)

Lois Holloway (Australia)

Yaoxiong Huang (China)

Sunny Jansen (USA)

Peter Johnston (Australia)

Bradley Kemp (USA)

Eddie (Yin) Khee (Singapore)

Stephen Kry (USA)

John Lawson (Australia)

Seok Lew (USA)

Ewa Majchrzak (Poland)

Eirik Malinen (Norway)

Aya Matsuyama (Australia)

Jagan Mazumdar (Australia)

Helen Mcaneney (UK)

Kumal Mitra (USA)

Oliver Morrish (UK)

Kwan Hoong Ng (Malaysia)

Graeme O'Keefe (Australia)

Ian Olver (Australia)

Trish Ostwald (Australia)

Ian Parkinson (Australia)

Napapong Pongnapang (Thailand)
Ramachandran Prabhakar (India)

Graham Rathbone (Australia)

Paul Reich (Australia)

Alistair Ross

Anatoly Rozenfeld (Australia)

Theo Samaras (Greece)

Sebastian Schostek (Germany)

I Sechopoulos (USA)

Francisco Sepulveda (UK)

D. Shepherd (UK)

Chengyu Shi (USA)

Ian Smith (Australia)

Natalka Suchowerska (Australia)

Zeeshan Syed (USA)

Cinzia Talamonti (Italy)

Alrefae Tareq (Kuwait)

D. N. Tarnita (Romania)

Michael Taylor (Australia)

Fergus Thomson (New Zealand)

Ana Luisa Trejos (Canada)

Frederica Vatta (Italy)

Sarah Way (USA)

Markus Wendling (Netherlands)

Luke Wilkinson (Australia)

Tony Wong (USA)

Ming-Chya Wu (Taiwan)

Zhaohua Wu (USA)

Shan Yau (Australia)

Yingchun Zhang (USA) 